\title{
Super-redes semicondutoras: Um laboratório de Mecânica Quântica
} (Semiconductor superlattices: A Quantum Mechanics laboratory)

\author{
Adenilson J. Chiquitd 1 e Francesco Lanciotti Jr. \\ Laboratório de Semicondutores, Departamento de Física, Universidade Federal de São Carlos, São Carlos, SP, Brasil \\ Recebido em 10/10/2003; Revisado em 02/06/2004; Aceito em 10/09/2004
}

\begin{abstract}
Neste trabalho, são discutidas algumas idéias relacionadas às alterações causadas na estrutura eletrônica de materiais semicondutores devido à presença de defeitos e desordem e suas conseqüências para a distribuição de portadores nestas estruturas. Em seguida, são apresentadas algumas das praticamente infindáveis aplicações das super-redes semicondutoras, agora sob um novo aspecto: verdadeiros laboratórios onde propriedades como a desordem causada por defeitos podem ser simuladas e estudadas sistematicamente.
\end{abstract}

Palavras-chave: super-redes, Mecânica Quântica.

In this work, some ideas related to the modifications on the electronic structure of semiconductor materials caused by defects and disorder are discussed. The modifications on the distribution of carriers are discussed as well. Next, we present some applications of the semiconductor superlattices, now focusing on microscopy laboratories, where properties like disorder induced by defects can be systematically studied.

Keywords: superlattices, Quantum Mechanics.

\section{Introduçãa}

Defeitos sempre existem em qualquer material e os semicondutores não fogem à regra. Um defeito pode ser originado de diversas maneiras, seja por uma alteração numa região de uma amostra causada por uma modificação na rede cristalina, seja pela adição de átomos estranhos como quando uma amostra passa por um processo de dopagem, por exemplo. Normalmente, quando se fala em defeitos em um cristal imaginase que somente mudanças não desejáveis nas características do material devem ser esperadas. Por um lado, esta idéia é correta pois os defeitos podem causar alterações pelas quais as características de um material podem ser totalmente alteradas e mesmo controladas pela presença de defeitos. Um exemplo disso é a localização de parte dos portadores (elétrons ou bura$\cos$ ), diminuindo a condutividade esperada de um sistema. Esta é uma consequiência realmente séria quando se fala na produção de dispositivos eletrônicos.

Pode-se considerar um efeito imediato da presença de defeitos em uma rede cristalina a introdução de uma desordem aleatória na estrutura. Podemos interpretar a desordem estudando uma série de fenômenos, mas vamos olhar aqui para os efeitos relacionados aos processos de condução de portadores. De uma maneira geral, a suposição de que uma rede cristalina é perfeitamente periódica é o ponto fundamental para o estudo de suas propriedades eletrônicas, mecânicas, de transporte, ópticas e assim por diante. Na realidade, o conceito de bandas de energia tem como base fundamental a periodicidade da rede cristalina e daí surgem conceitos como o da massa efetiva e intervalos de energias proibidas (ou gap, discutido nas seções seguintes), por exemplo. A presença de defeitos provoca a quebra da propriedade simétrica da periodicidade, introduz fontes de espalhamento, provocam também a quebra da coerência das funções de onda que descrevem o cristal (funções de Bloch) [1]. O problema está em como tratar a não-periodicidade para a determinação das propriedades do cristal e uma maneira de realizar esta tarefa é considerando a não periodicidade como apenas um perturbação ao sistema eletrônico, tendo

\footnotetext{
${ }^{1}$ Enviar correspondência para Adenilson J. Chiquito. E-mail: chiquito@df.ufscar.br.
} 
como efeito principal a transição entre vários estados coerentes.

Por outro lado, olhando com mais cuidado é fácil e interessante perceber que os efeitos da desordem nem sempre são ruins: os processos de espalhamento de portadores provocados pela presença de defeitos contribuem para a condutividade dc (corrente contínua). Aliás, o processo de dopagem de um cristal, bastante conhecido e usado em semicondutores, consiste basicamente na introdução de átomos estranhos à rede cristalina causando desordem na estrutura. Por outro lado, a introdução de dopantes em um cristal semicondutor determina o portador (elétron ou buraco) que será responsável pelo tipo de condutividade do cristal. A consequiência tecnológica imediata disto foi a construção de diodos, transistores, resultando na infinidade de dispositivos eletrônicos atualmente disponíveis. Mais um exemplo interessante é o caso dos pontos quânticos auto-organizados que se "auto-constroem" espontaneamente e por isso têm uma distribuição aleatória, produzindo um potencial também aleatório no qual portadores podem ficar presos. Dessa forma, pontos quânticos também podem ser vistos como defeitos e vêm sendo exaustivamente estudados com o objetivo de construção de transistores e laseres de alta eficiência [2].

Seguindo com mais exemplos, a presença da desordem causada pelos defeitos é fundamental para vários processos na Natureza como a observação do Efeito Hall Quântico Inteiro [3]. É indispensável a consideração da existência de desordem para a explicação do efeito: como resultado da desordem, os níveis de Landau2 2 sofrem um alargamento o que pode permitir a localização de elétrons e assim, mesmo quando o nível de Fermi está entre dois níveis de Landau, tem-se a contribuição dos estados localizados (portadores estão presos!) que aceitam elétrons. Mas como estados localizados não contribuem para a condutividade, observam-se os conhecidos patamares constantes de condutividade/resistividade.

No que segue, serão discutidas algumas alterações causadas pela desordem em estruturas semicondutoras e suas consequiências, por exemplo, para a condutividade. Em seguida, apresentamos idéias a respeito de como uma heteroestrutura semicondutora pode ser usada no controle e estudo da desordem causada por alterações estruturais. Será sempre considerado que os portadores de carga são os elétrons, uma vez que um tratamento análogo pode ser feito para buracos.

\section{Alguns efeitos da desordem em cristais}

Sempre que estudamos as propriedades de um sistema qualquer, é importante considerar condições de simetria, as quais geralmente tornam o trabalho de descrição do sistema mais simples. No caso de um sólido, a presença de uma regularidade na disposição dos átomos na rede cristalina é uma peça chave para a descrição destes sistemas. Na realidade foi esta peculiaridade que permitiu o desenvolvimento da teoria de bandas cristalinas a qual tem como ponto fundamental a periodicidade da rede e por conseqüência a das funções de Bloch [1].

A presença de defeitos em um sólido pode quebrar a periodicidade cristalina. Em situações onde o número de defeitos é pequeno de tal forma que a rede cristalina sofra apenas uma perturbação, os processos de transporte de portadores não são fortemente alterados e ainda são determinados pelos estados estendidos definidos pelas funções de Bloch ao longo de todo o cristal. Essencialmente, os defeitos estão localizados em regiões bem específicas do material e podem ou não interagir e de forma geral são descritos por funções de onda locais (estados localizados). Por outro lado, quando o número de defeitos aumenta, a desordem introduzida também aumenta e estados localizados e estendidos misturam-se fortemente dando origem a um espectro contínuo de energias.

Em 1958 P. W. Anderson [4], estudando os efeitos da presença de desordem em um cristal mostrou que a distribuição e evolução dos estados localizados produzidos pelo aumento da desordem podem ser esquematizados como na Fig. 1(a). As energias que separam os estados localizados dos estados estendidos, mostradas na figura ( $E_{1}$ e $\left.E_{2}\right)$, são definidas considerando que a condutividade dos estados localizados vai a zero em baixas temperaturas. Num tratamento mais rigoroso, as energias $E_{1}$ e $E_{2}$ dependem da dimensionalidade do sistema, mas este assunto foge ao escopo deste trabalho. Aos leitores interessados, a Ref. [5] trata formalmente esta particularidade de uma maneira clara, porém bastante técnica. Como ficam então, as propriedades de transporte de cargas quando

\footnotetext{
${ }^{2}$ O problema de elétrons em um campo magnético pode ser estudado considerando a energia magnética na equação de Schrödinger. Supondo, por exemplo, que o campo magnético esteja na direção $z$, o movimento eletrônico no plano $x-y$ será dado por um hamiltoniano semelhante ao de um oscilador harmônico, cujas soluções levam a estados quantizados, conhecidos como níveis de Landau.
} 
se fala em estados estendidos e localizados? De uma forma simples espera-se que sejam bastante diferentes: em um estado localizado os elétrons estão presos numa determinada região do cristal e não se acoplam com outros estados eletrônicos. A única forma pela qual um elétron pode mover-se através de estados localizados é via processos como tunelamento ou hopping (que literalmente significa que os elétrons saltam de um para outro estado eletrônico). A condução eletrônica via hopping é na verdade essencial para o transporte em materiais caracterizados por estados localizados. Existem três mecanismos de condução nos quais os elétrons saltam entre estados eletrônicos [Fig. 1(b)]: a) ativação térmica, através da qual elétrons localizados saltam para uma região de estados estendidos e aí participam dos processos de condução do material; b) ativação térmica entre estados espacialmente próximos, entre os quais há a superposição das funções de onda que os descrevem; c) os elétrons saltam para um estado vizinho não necessariamente mais próximo espacialmente, mas para aquele que tem uma energia característica mais próxima [6]. Estes três processos de condução podem ser matematicamente expressos pela condutividade como:

$$
\begin{aligned}
& \text { processo }(a) \rightarrow \sigma=\sigma_{0} \exp \left(\frac{-E}{k T}\right) \\
& \text { processo }(b) \rightarrow \sigma=\sigma_{0} \exp \left(-\alpha R-\frac{E}{k T}\right) \\
& \operatorname{processo}(c) \rightarrow \sigma=\sigma_{0} \exp \left(\frac{-T_{0}}{T}\right)^{1 / 4},
\end{aligned}
$$

onde $\sigma_{0}, \alpha, T_{0}$ são constantes, $k$ é a constante de Boltzmman, $E$ é a energia envolvida nos processos de ativação térmica e $R$ é a distância de hopping.

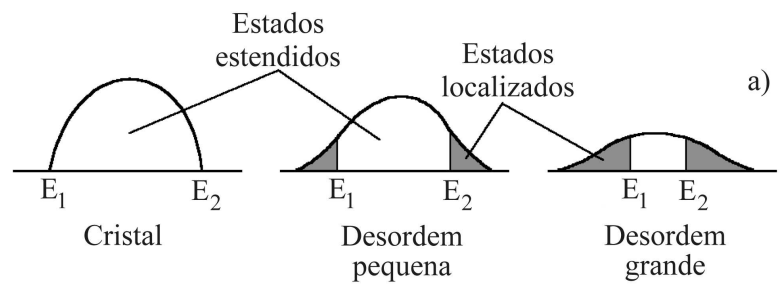

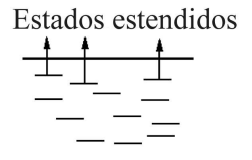

Ativação térmica

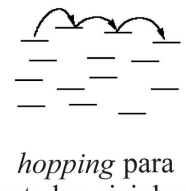

estados vizinhos b)

Figura 1 - (a) distribuição e evolução dos estados localizados produzidos pelo aumento da desordem no modelo de Anderson; (b) mecanismos de condução possíveis para materiais onde há localização de elétrons.
Como comparação, a equação seguinte mostra a condutividade esperada em um dado material seguindo o modelo clássico de Drude [1]:

$$
\sigma=\frac{n e^{2} \tau}{m}
$$

onde $\mathrm{n}$ é número de elétrons, e a carga eletrônica, $\mathrm{m}$ a massa dos elétrons e $\tau$ é o tempo entre colisões em que o elétron viaja sem sofrer choques (supostos elásticos). Note que não há dependência explícita na temperatura na Eq. (4) e esta equação vale na situação de equilíbrio termodinâmico em uma dada temperatura do sistema estudado.

A descrição acima não pretende ser completa em todos os detalhes que envolvem os mecanismos de condução entre os estados localizados, mas apenas mostrar ao leitor que realmente a presença de localização altera fortemente a forma de condução eletrônica esperada em um dado material. O estudo dos efeitos da desordem podem ser realizados através de técnicas experimentais nas quais são observadas as características do transporte de corrente ou as características ópticas do sistema. As consequiências para as propriedades de uma estrutura onde há desordem são muito variadas e levam a muitos resultados interessantes e às vezes surpreendentes [7]. O grande problema entretanto, é que um estudo sistemático dos efeitos da desordem fica algo complicado quando não se tem controle sobre o parâmetro mais importante da investigação que é a própria desordem. Na seção seguinte vamos tratar algumas propriedades apresentadas por super-redes artificiais, nas quais a desordem pode ser controlada de uma maneira bem simples. Nelas, iremos tratar a idéia de localização de cargas devido à alterações induzidas na estrutura mostrando o efeito da desordem em um sistema eletrônico.

\section{Super-redes - Controle artificial da estrutura eletrônica}

De uma maneira geral, não é uma tarefa simples tratar os efeitos da desordem em sistema. Os materiais semicondutores altamente dopados foram alvo de um grande número de trabalhos científicos em busca da compreensão e estudo dos efeitos da desordem nas propriedades destes materiais [7]. Estes sistemas foram escolhidos porque a incorporação de átomos dopantes introduz uma desordem aleatória no sistema. Quanto maior for a dopagem, maior será a desordem mas 
também será maior o número de elétrons livres no sistema, aumentado a interação coulombiana no sistema. Assim, introduz-se também uma dificuldade para o estudo dos efeitos da desordem nestes sistemas, uma vez que é difícil a separação entre as alterações provocadas pela desordem devido à presença de átomos dopantes e pela alta densidade eletrônica que também causa desordem.

Desde o surgimento e mais acentuadamente depois do aprimoramento (décadas de 70 e 80 , respectivamente), a técnica de crescimento de cristais atomicamente controlados através de MBE (Epitaxia por Feixes Moleculares) mostra-se altamente eficaz na construção de estruturas artificiais com aplicações para os mais diversos fins: desde estudos básicos em Física Quântica, até o desenvolvimento de estruturas complexas para novos dispositivos eletrônicos. Uma das estruturas mais estudadas crescidas por MBE são as super-redes. Uma super-rede semicondutora é formada pelo crescimento periódico e alternado de camadas (espessura $\approx 10-100 \mathrm{~nm}$ ) de dois semicondutores com energias de $g a p \sqrt[3]{3}$ diferentes, como mostra esquematicamente a Fig. 2(a). Como têm energia de gap diferentes, deve aparecer uma descontinuidade de energia nas interfaces entre duas camadas adjacentes [Fig. 2(b)]. Note que as alterações são produzidas em apenas uma das direções espaciais (direção $z$ na figura).

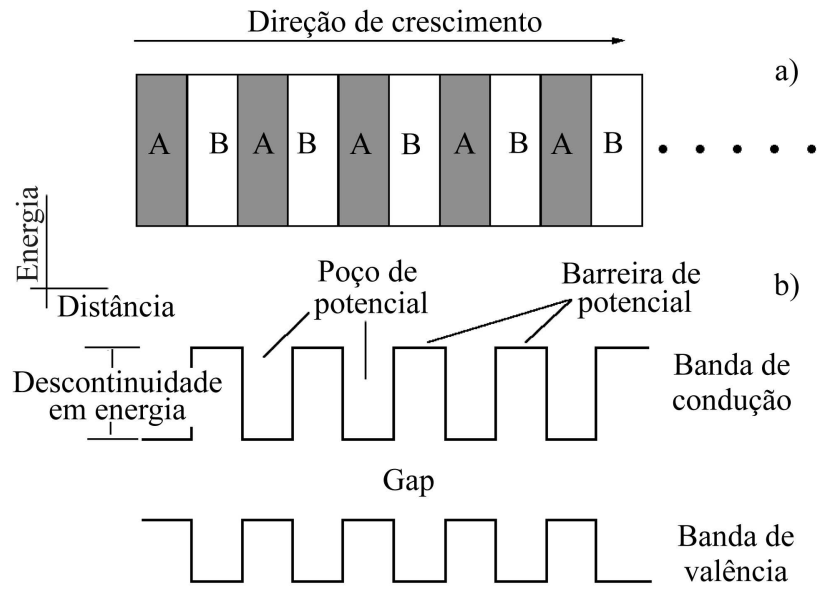

Figura 2 - Super-redes crescidas por MBE: (a) uma super-rede é formada pelo crescimento periódico e alternado de camadas de dois semicondutores com energias de gap diferentes; (b) tendo energias de gap diferentes, há uma descontinuidade em energia nas interfaces entre camadas adjacentes. Como referência foram colocados eixos coordenados (energia e distância) no lado esquerdo da figura.

É interessante aqui fazer uma comparação entre uma super-rede e uma rede cristalina (por exemplo, do material $\mathrm{A}$ ou $\mathrm{B}$ que formam a super-rede). Observando a Fig. 2(b) pode-se notar a formação de poços de potencial separados por barreiras de maneira análoga ao que acontece com o potencial periódico num cristal natural. Nesta comparação, podemos dizer que os poços de potencial comportam-se como macro-átomos numa rede cristalina com um distanciamento maior (esta distância, em comparação com uma rede cristalina natural, é análoga ao parâmetro de rede que mede a distância entre dois átomos) e daí vem o nome super-redes cristalinas. Intuitivamente, é possível supor que da mesma maneira que a disposição periódica dos átomos numa rede cristalina origina estados eletrônicos estendidos por todo o cristal, a disposição também periódica dos poços na super-rede origine estados do mesmo tipo. $\mathrm{Na}$ realidade a afirmação anterior pode ser provada facilmente, calculando-se a estrutura eletrônica de uma super-rede [8] pela qual observa-se a criação de estados eletrônicos estendidos na direção de crescimento da super-rede, conhecidos por minibandas. Uma indicação de como isso pode ser realizado será dada mais adiante.

Quando as dimensões de um sistema são comparáveis ao comprimento de onda de De Broglie $\left(\lambda_{B}\right)$, o movimento dos portadores neste sistema tornase quantizado, implicando em mudanças no espectro energético e nas propriedades dinâmicas dos portadores. Se somente uma das dimensões é comparável a $\lambda_{B}$, diz-se que o sistema comporta-se dinamicamente como um sistema bidimensional (2D, poços quânticos). Limitando o movimento em duas e depois três direções, serão obtidos sistemas unidimensionais (1D, fios quânticos) e zero-dimensionais (0D, pontos quânticos). Por esta regra, como somente na direção de crescimento das super-redes temos comprimentos comparáveis com $\lambda_{B}$, pode-se dizer que uma super-rede é unidimensional. Num tratamento mais geral, as super-redes são na realidade tridimensionais pois as alterações que ocorrem apenas na direção de crescimento de uma amostra afetam tanto a estrutura eletrônica nesta direção como também nas outras duas, como mostraremos adiante.

Agora, lembrando que as super-redes são artificiais, podemos alterá-las da forma que for conveniente para a realização de um determinado estudo de interesse. Por exemplo, podemos aumentar ou

\footnotetext{
${ }^{3}$ Gap de energia ou intervalo de energia proibida refere-se ao intervalo de energia que separa o máximo da banda de valência de um cristal do mínimo da banda de condução do cristal. Neste intervalo, em princípio, não existem níveis de energia acessíveis aos elétrons.
} 
diminuir a espessura de uma barreira ou poço de potencial, permitindo ou não interação entre elétrons em camadas adjacentes; podemos alterar a espessura total da amostra, apenas crescendo mais ou menos camadas; podemos variar o número de elétrons na estrutura, controlando a dopagem, e assim por diante. Temos em mãos um verdadeiro laboratório de Mecânica Quântica, no qual podemos estudar e controlar a interação entre as funções de onda que descrevem os elétrons nos poços de potencial; podemos estudar como a existência de uma faixa de energia (minibanda) afeta os fenômenos de transporte de cargas através da estrutura, como as propriedades ópticas são alteradas e assim por diante, apenas construindo estruturas diferentes. Para mostrar a relação entre a estrutura cristalina e eletrônica numa super-rede, vamos antes calcular as energias e funções de onda em um poço de potencial formado por dois materiais semicondutores diferentes (podemos considerar este sistema um "átomo" da um super-rede). Para não sobrecarregar a Fig. 2, esta discussão será baseada na Fig. 3.

O comportamento dos elétrons neste sistema é descrito pela equação de Schrödinger,

$$
\left[\frac{-\hbar^{2}}{2 m^{*}} \nabla^{2}+V(z)\right] \Psi(x, y, z)=E \Psi(x, y, z) .
$$

$E$ é a energia total do nível quantizado e $\Psi(x, y, z)$ é a função de onda associada. Supondo que o problema pode ser desacoplado nas direções $x, y$ e $z$, a energia correspondente a um dado estado nesta estrutura é descrita por

$$
E_{n}=E_{n z}+\frac{\hbar^{2}}{2 m^{*}}\left(k_{x}^{2}+k_{y}^{2}\right),
$$

onde o primeiro termo é relativo à energia dos níveis discretos na direção $z$. O outro termo descreve a dispersão parabólica da energia nas direções $x$ e $y$. Associada a esta energia está a função de onda

$$
\Psi(x, y, z)=f(z) e^{i k_{x} x} e^{i k_{y} y},
$$

onde $f(z)$ é a função de onda que descreve o movimento na direção $z$, enquanto que as exponenciais descrevem o movimento quase livre dos portadores nos planos $x$ e $y$. Observe que a solução para a Eq. (5), dada pela Eq. (7), está escrita como o produto de três funções independentes nas direções $x, y$ e $z$. A energia descrita por $E_{n z}$ é a energia de confinamento para as partículas no poço de potencial e é uma consequiência direta do princípio da incerteza: quando uma partícula é confinada no espaço real [dentro de uma distância $L$, Fig. 3(a)], a incerteza na componente $z$ do momento da partícula aumenta por uma quantidade $\hbar / L$. O correspondente aumento na energia é dado por $E_{n z}$.

Quando poços quânticos como os descritos acima são colocados lado a lado, obtém-se uma super-rede como mencionado acima. Se a largura das barreiras é suficientemente pequena haverá a sobreposição das funções de onda que descrevem os portadores em poços quânticos vizinhos [Fig. 3(b)]. Inicialmente degenerados, quando em poços quânticos isolados, os estados discretos se alargam em bandas permitidas (minibandas) separadas por faixas de energias proibidas (minigaps), como em uma rede cristalina natural. Dessa forma, cada estado $E_{n z}$ [Eq. (6)] de um poço quântico alarga-se em um minibanda $E_{n z}\left(k_{z}\right)$ na super-rede. Assim,

$$
E_{n}\left(k_{x}, k_{y}, k_{z}\right)=E_{n z}\left(k_{z}\right)+\frac{\hbar^{2}}{2 m^{*}}\left(k_{x}^{2}+k_{y}^{2}\right) .
$$
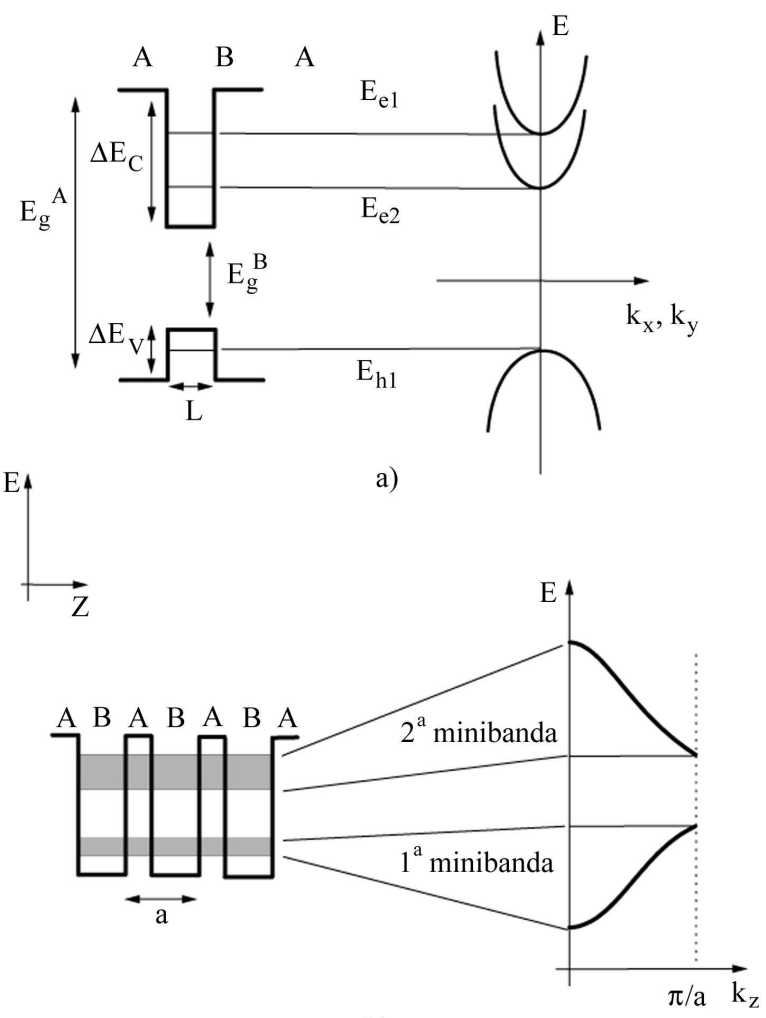

b)

Figura 3 - (a) Esquema de um poço quântico com largura $L$ e os níveis de energia discretos correspondentes. $\Delta E_{C}, \Delta E_{V}$ são as descontinuidades nos perfis das bandas de condução e valência causada pela diferença das energias dos gaps dos materiais A e B $\left(E_{g}^{A}\right.$ e $\left.E_{g}^{B}\right)$. No espaço $E \times k$, vê-se que para cada estado quantizado dentro do poço $\left(E_{e 1}, E_{e 2}\right.$ e $\left.E_{h 1}\right)$ há ainda uma dispersão parabólica para as partículas nas direções $x, y$. (b) super-rede de período $a$ e a estrutura de minibandas correspondente, no caso unidimensional. $\mathrm{O}$ alargamento dos níveis discretos origina minibandas com dispersões em $k_{z}, k_{x}$ e $k_{y}$ (não mostradas nestas duas últimas direções). 
A energia $E_{n z}\left(k_{z}\right)$, calculada usando um modelo simples como o método Tight-Binding [9] 1], tem a forma

$$
E_{n z}\left(k_{z}\right)=W\left[1-\cos \left(k_{z} a\right)\right],
$$

onde $W$ é a largura da minibanda e $a$ é o período da super-rede [Fig. 3(b)]. Distintamente dos poços quânticos, as super-redes apresentam uma dispersão em energia na direção $k_{z}$, ou seja, os estados eletrônicos nas super-redes são estendidos como num cristal natural.

Para ilustrar como mudanças na estrutura das super-redes podem alterar significativamente as propriedades apresentadas, temos na Fig. 3 exemplos de diferentes super-redes e o comportamento das funções de onda que descrevem sua estrutura eletrônica, obtidas via solução da Eq. (5). Na Fig. 4(a), temos o caso de uma super-rede na qual o espaçamento entre os poços de potencial é muito grande para que haja interação entre os elétrons localizados em poços adjacentes e assim, são descritos por funções de onda localizadas em cada poço. Na Fig. 4(b), diminuiu-se a distância entre os poços e pode-se imediatamente observar que a interação entre os estados eletrônicos é dominante. Nesse caso não se pode falar sobre a localização de elétrons nos poços mas sim, numa distribuição aproximadamente uniforme através de toda a estrutura dando origem a um contínuo de estados. Na Fig. 4(c) está o caso mais interessante para o nosso estudo, na qual aparece uma variação da largura dos poços enquanto que a largura das barreira permaneceu constante. Note o comportamento das funções de onda: em regiões onde a periodicidade da super-rede foi quebrada, elétrons ficam localizados. Fica fácil observar porque o uso de uma super-rede pode oferecer vantagens o estudo da desordem, uma vez que podemos em princípio estimar a intensidade da desordem: em relação a uma estrutura ordenada, a variação artificial das larguras dos poços de potencial leva a uma distribuição de energias, que mede o grau da desordem induzida artificialmente. E, separadamente, os efeitos da dopagem podem ser controlados pois a técnica de crescimento (MBE) permite que a dopagem seja controlada independentemente do processo de formação do cristal.

É interessante notar que neste exemplo apenas foram introduzidos alguns poços com larguras diferentes, não se configurando portanto uma desordem aleatória como deve ser esperado num cristal natural. Esta situação pode ser conseguida usando uma estru- tura maior, com muitos períodos [10]. Mas mesmo na situação simples apresentada aqui, observa-se que a quebra de coerência na estrutura é responsável por uma drástica alteração na distribuição eletrônica na superrede.
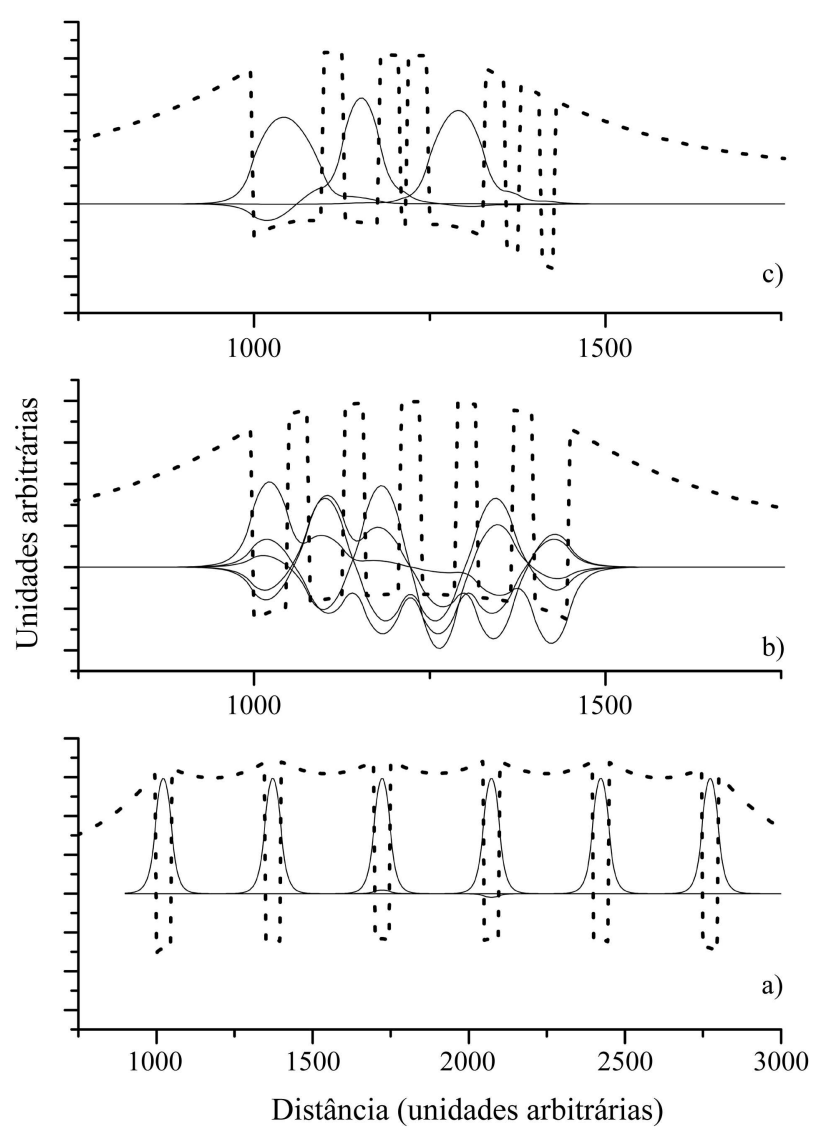

Figura 4 - Banda de condução (linha pontilhada) e probabilidades (linha contínua) para três tipos de super-redes: (a) super-rede na qual o espaçamento entre os poços de potencial é muito grande para que haja interação entre os elétrons localizados em poços adjacentes; (b) diminuiu-se a distância entre os poços e pode-se imediatamente observar que a interação entre os estados eletrônicos é dominante; (c) variação "aleatória" da largura dos poços enquanto que a largura das barreira permaneceu constante.

Voltemo-nos então, para uma aplicação de nosso laboratório de Mecânica Quântica numa situação real. Como um exemplo, na Fig. 5(a) temos uma curva de distribuição de elétrons em uma super-rede construída com introdução de desordem aleatória durante o crescimento e com dopagem uniforme para todas as camadas. A super-rede usada é formada por $40 \mathrm{a}$ 50 camadas de GaAs (arsenieto de Gálio) e AlGaAs (Alumínio-arsenieto de Gálio) e foi crescida em uma máquina de MBE no Instituto de Física de São Carlos (USP). Como era esperado pela presença de desordem, os elétrons não estão distribuídos uniformemente ao longo da estrutura, mas apresentam uma "modulação" 
indicando o efeito da desordem na estrutura da superrede. A curva de distribuição eletrônica apresentada foi determinada experimentalmente através de medidas de capacitância [11, 12]. Resumidamente, a distribuição experimental pode ser assim determinada: deposita-se uma camada de metal (geralmente $\mathrm{Au}$ ) sobre a superrede e mede-se a capacitância diferencial da junção metal-semicondutor (MS) formada em função de diferentes voltagens aplicadas ao sistema. Pode-se encontrar o perfil de distribuição de elétrons com ajuda das seguintes equações [13]:

$$
\begin{gathered}
n(w)=\frac{C^{3}}{q S \varepsilon_{s}}\left(\frac{d C}{d V}\right)^{-1} \\
w=\frac{\varepsilon_{s}}{C} S
\end{gathered}
$$
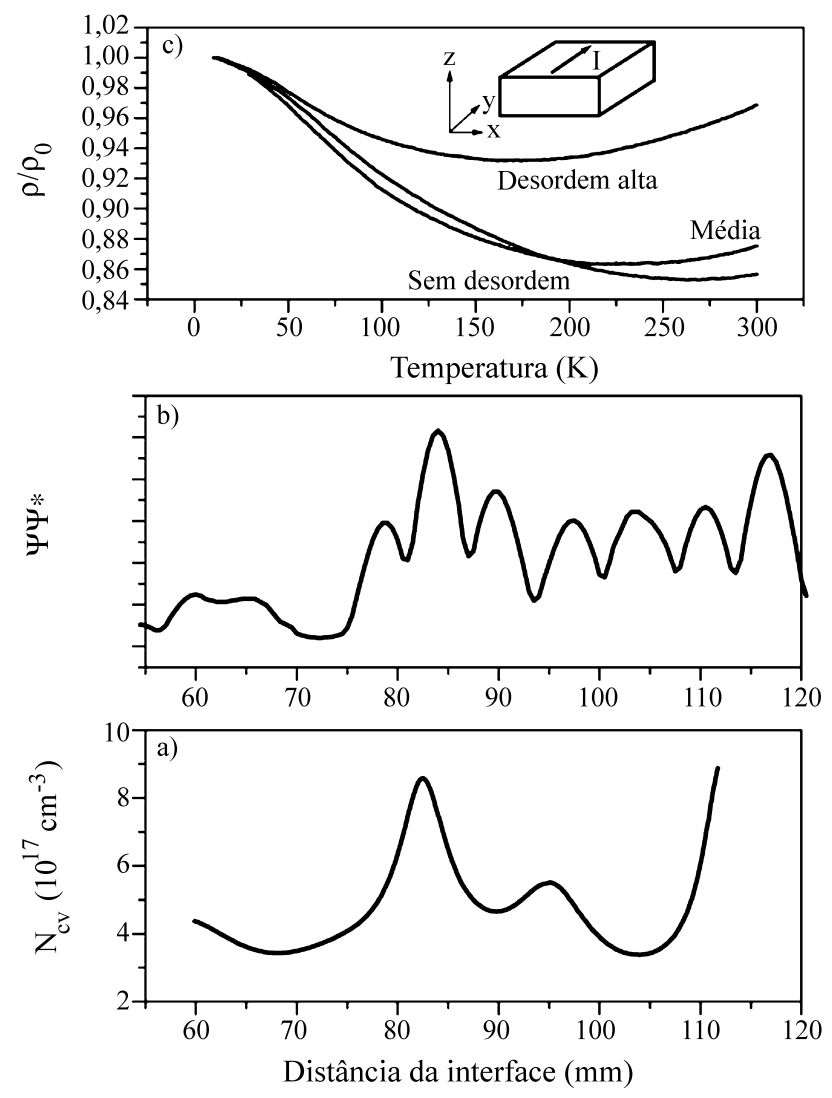

Figura 5 - (a) curva de distribuição de elétrons em uma superrede construída com introdução de desordem aleatória durante o crescimento e com dopagem uniforme para todas as camadas; (b) probabilidade de encontrar elétrons em função da distância que é maior nas regiões onde há quebra de periodicidade da super-rede; (c) variação da resistividade paralela como função da temperatura em amostras com diferentes desordens induzidas. No detalhe vê-se um diagrama mostrando como os dados apresentados em c) foram obtidos. Note que as medidas foram realizadas numa direção perpendicular $(y)$ à direção de crescimento $(z)$. onde $n(w)$ é a concentração eletrônica na posição $w$ medida a partir da interface MS, $C$ é a capacitância, $S$ a área do contato Schottky. Com estas duas equações podemos determinar qual é a concentração de elétrons num determinado ponto da estrutura $(w)$ e qual é a distância deste ponto em relação à interface MS.

Justamente nas posições onde foram alteradas intencionalmente as larguras dos poços de potencial aparecem picos no perfil de distribuição de elétrons, mostrando localização de portadores como era o esperado. Os cálculos referentes à super-rede em questão estão colocados na Fig. 5(b). Note a concordância com a discussão acima: a probabilidade de encontrar os elétrons na estrutura é maior nas regiões onde há localização de elétrons $(80 \mathrm{~nm})$. Deve-se comparar com cuidado as Fig. 5(a) e Fig. 5(b): os máximos na probabilidade em 65 e $105 \mathrm{~nm}$ não aparecem no perfil de distribuição de elétrons experimental porque o campo elétrico aplicado para a determinação do perfil não foi levado em conta nos cálculos. Além disso, o perfil de distribuição derivado da capacitância apresenta uma limitação quando a dopagem varia acentuadamente em regiões adjacentes. Neste caso, aparecem vales [como entre 60 e $75 \mathrm{~nm}$ na Fig. 5(b)] antes de um pico [14].

De uma forma simples, pode-se ver que as superredes com desordem induzida permitem a observação de propriedades pertinentes à localização de cargas e que a manipulação desta desordem artificial pode permitir uma gama enorme de diferentes acoplamentos entre estados eletrônicos em poços vizinhos. Finalmente, para mostrar o caráter tridimensional do sistema, alguns experimentos de transporte eletrônico foram realizados em amostras com diferentes desordens induzidas como mostrado na Fig. 5(c) para estudos de interferência quântica [15, 10]. Nestes experimentos, determinou-se a variação da resistividade nas direções perpendiculares à direção de crescimento das amostras, ou seja, no plano da super-rede [veja o detalhe destacado na Fig. (5)]. Apesar da desordem estrutural ter sido limitada à direção do crescimento das estruturas, pode-se notar que o transporte de carga no plano das camadas (perpendicular à direção de crescimento) também foi afetado como mostra a Fig. 5(c): observe a diferença entre os comportamentos apresentados pela resistividade em função da temperatura, mostrando que a desordem tem uma contribuição significativa para o transporte de corrente através da estrutura, localizando cargas em determinadas regiões na direção de crescimento e alterando os processos de condução também 
nas outras duas direções.

\section{Conclusão}

Neste artigo foram apresentadas algumas idéias a respeito dos efeitos da desordem em um cristal e a possibilidade de estudá-los usando uma estrutura artificial como uma super-rede semicondutora. As idéias abordadas aqui, principalmente com respeito à manipulação da estrutura de uma super-rede, mostram uma aplicação prática de conceitos dados em cursos introdutórios de Física Moderna, nos quais estudam-se os efeitos para o movimento de elétrons entre poços e barreiras de potencial. Os autores agradecem ao Prof. Yuri A. Pusep (Grupo de MBE, IFSC-USP) pelas amostras cedidas e discussões.

\section{Referências}

[1] C. Kittel, Introduction to Solid State Physics (John Wiley and Sons, New York, 1968), 3rd. ed.

[2] A.J. Chiquito, Rev. Bras. Ens. Fís. 23, 159 (2001).

[3] K. von Klitzing, G. Dorda and M. Pepper, Phys. Rev. Lett. 45, 494 (1980).

[4] P.W. Anderson, Phys. Rev. 109, 1492 (1958).
[5] E. Abrahams, P.W. Anderson, D.C. Licciardello and T.V. Ramakrishnan, Phys. Rev. Lett. 42, 673 (1979).

[6] N.F. Mott, J.Non-Cryst.Solids 1, 1 (1968).

[7] I. Shklovskii and A.L. Efros, Electron properties of Doped Semiconductors (Springer-Verlag, 1984); V. Ambegaokar, B.I. Halperin and J.S. Langer, Phys. Rev. B 4, 2612 (1971); M.A. Pollak, J. Non-Cryst. Solids 11, 1 (1972); S. Kirkpatrick, Phys. Rev. Lett. 27, 1722 (1971); C.H. Seager and G.E. Pike, Phys. Rev. B 10, 1435 (1974).

[8] C. Weisbuch and B. Vinter, Quantum Semiconductor Structures (Academic Press, San Diego, 1991).

[9] N.W. Ashcroft and N.D. Mermin, Solid State Physics (Saunders College Pub., Fort Worth, 1976).

[10] A.J. Chiquito, Yu. A. Pusep, G.M. Gusev and A.I. Toropov, Phys. Rev. B 66, 035323 (2002).

[11] E.H. Rhoderick and R.H. Williams, Metal Semiconductor Contacts (Oxford, 1988).

[12] P. Blood, Semicond. Sci. Technol. 1, 1 (1986).

[13] W.C. Johnson and P.T. Panousis, IEEE Trans. Elect. Devices ED-18, 965 (1971).

[14] H. Kroemer and W-Y. Chien, Solid State Electron. 24, 655 (1981).

[15] Yu. A. Pusep, A.J. Chiquito, S. Mergulhão and A.I. Toropov, J. Appl. Phys. 92, 3830 (2002). 